\title{
THE KINETICS AND MECHANISM OF OXIDATION OF REDUCED SPINACH FERREDOXIN BY MOLECULAR OXYGEN AND ITS REDUCED PRODUCTS
}

\author{
BARBARA HOSEIN * and GRAHAM PALMER \\ Department of Biological Chemistry and Biophysics Research Division, University of Michigan, Ann Arbor, MI 48103 and the Department \\ of Biochemistry, Rice University, P.O. Box 1892 Houston, TX 77251 (U.S.A.)
}

(Received December 6th, 1982)

(Revised manuscript received March 23rd, 1983)

Key words: Ferredoxin; Superoxide anion; Hydrogen peroxide; Oxygen; (Spinach)

Reduced spinach ferredoxin reacts with molecular oxygen in an autocatalytic reaction characterized by a hyperbolic dependence on oxygen concentration. The kinetics of the reaction indicate formation of a reduced ferredoxin-oxygen intermediate complex and production of superoxide anion which may also react with reduced ferredoxin. Hydrogen peroxide, which is formed from superoxide, in turn reoxidizes reduced ferredoxin at a rate nearly 10-times faster than that of the comparable reaction with oxygen. The kinetics of reaction of hydrogen peroxide with reduced ferredoxin are biphasic. The substrate dependence of the first phase of the reaction is consistent with a simple one-step equilibrium reaction. The second phase of the reaction could be eliminated by addition of the radical trapper, sodium formate.

\section{Introduction}

The iron-sulfur site of the nonheme iron protein spinach ferredoxin has been shown to react with oxygen under a variety of in vitro conditions. For example, it has been demonstrated that spinach ferredoxin reduced by illuminated chloroplasts can be reoxidized in the dark with the uptake of one equivalent of oxygen per four equivalents of ferredoxin [1], consistent with the one-electron character of this iron-sulfur protein [2]. The ultimate product of the reduction of oxygen must therefore be water. Arnon et al. [3] have proposed that the mechanism of noncyclic photophosphorylation in the absence of $\mathrm{NADP}^{+}$involves the photoreduction of ferredoxin and its reoxidation by oxygen, while Telfer et al. [4] have determined that hydro-

\footnotetext{
* Present address: The Lindsley F. Kimball Research Institute of The New York Blood Center, New York, NY 10021, U.S.A.
}

gen peroxide is a primary product of the reoxidation reaction in illuminated chloroplasts and in solution. Using sensitive chemical techniques, Misra and Fridovich [5] demonstrated that superoxide is produced by the reoxidation of both bacterial and spinach ferredoxins reduced by NADPH plus ferredoxin-NADP reductase, with which ferredoxin forms a $1: 1$ complex [6]. Using a similar system, Nakamura and Kimura [7] showed that the production of superoxide anion was a result of direct electron transfer from ferredoxin to molecular oxygen, and not from the flavoprotein to oxygen. These authors suggested an analogy between their system and complex proteins such as xanthine oxidase, arguing that the production of superoxide anion by xanthine oxidase is due to the iron-sulfur center rather than the flavin moiety; however, this viewpoint is opposed to current understanding of the mechanism of action of xanthine oxidase which has been characterized in detail $[8,9]$. Allen [10] has shown that superoxide dis- 
mutase inhibited ferredoxin-mediated photosynthetic uptake of oxygen in broken chloroplasts and gave preliminary evidence that superoxide dismutase inhibited the rate of oxidation by oxygen of chemically reduced ferredoxin.

We have examine in detail the kinetics of reoxidation of chemically reduced ferredoxin in the absence of potentially complicating components such as NADPH and ferredoxin $\mathrm{NADP}^{+}$reductase. We find that reduced ferredoxin reacts not only with molecular oxygen, but also even more vigorously with the intermediates of the reoxidation reaction, namely, hydrogen peroxide and possibly superoxide anion. These species have been shown to be present in chloroplasts [11], and thus plant ferredoxin may serve a secondary function as a backup radical scavenger in the absence of more effective dismutases and catalases.

\section{Materials and Methods}

Spinach ferredoxin was purified according to the procedure of Petering and Palmer [12]. Ferredoxin concentrations were determined spectrophotometrically using an extinction coefficient at $420 \mathrm{~nm}$ of $9400 \mathrm{M}^{-1} \cdot \mathrm{cm}^{-1}$. Purity was characterized by the value of the ratio of the absorbance of ferredoxin at $420 \mathrm{~nm}$ to that at 275 $\mathrm{nm}$; the highest reproducible value was 0.48 .

Catalase (Grade A) was from Calbiochem and horseradish peroxidase (type VI) was from Sigma. Superoxide dismutase was a gift from Dr. David Lambeth. Sodium dithionite came from Hardman and Holden.

All solutions were prepared in $0.15 \mathrm{M}$ Tris- $\mathrm{HCl}$ buffer. Solutions in glass tonometers were made anaerobic by equilibrating the liquid phase at least six times with an atmosphere of nitrogen gas purified by passage over hot copper turnings. The oxygen concentration of the nitrogen treated in this way was reduced to less than $0.1 \mathrm{ppm}$ [13].

The stopped-flow instrument used was described by Ballou [13] as modified by Lambeth and Palmer [14]. Reactions were monitored at 420 $\mathrm{nm}$ using a Kodak infrared rejection filter plus Corning No. 5030 filter, and a spectral bandwidth of $3 \mathrm{~nm}$ was used. The stopped-flow instrument had a $20 \mathrm{~mm}$ path length, a measured dead time of $1.3 \mathrm{~ms}$ and an overall instrumental time constant of $0.2 \mathrm{~ms}$. All experiments were conducted at $25^{\circ} \mathrm{C}$, and reactants were mixed in a ratio of $1: 1$ by volume. Concentrations after mixing are given.

Absorbance signals from the photometer were amplified to an optimal value and recorded with a Varian C-1024 time average computer (CAT) with the slower phase of reaction measured with a Y-T recorder. Plots testing for first-order behavior of kinetic traces on stopped-flow experiments were obtained by postprocessing with a logarithmic amplifier. Experiments were generally designed to give pseudo-first-order kinetics by having one reactant at least 5 -fold in excess.

Reduced ferredoxin solutions were prepared by the incremental addition of small portions of a concentrated sodium dithionite solution in an anaerobic tonometer equipped with a side-arm cuvette. The reductant was monitored at $420 \mathrm{~nm}$ and the addition was concluded just beyond the endpoint. The product of oxidation of dithionite, sulfite, is a poor reducing agent and is not oxidized by oxygen.

\section{Results}

The reoxidation of reduced spinach ferredoxin by oxygen proceeded with nominal half-lives ranging from 6 to $20 \mathrm{~s}$ over a 10-fold range of oxygen concentration $(0.065-0.65 \mathrm{mM})$. Fig. 1 shows reaction traces and semilogarithmic plots for the reaction of reduced ferredoxin with 0.13 (a) and 0.65 $\mathrm{mM}$ (c) oxygen. At the lower oxygen concentration a pronounced lag phase lasting for one half-life is seen, after which the reaction follows first-order kinetics for approximately two half-lives and then increases in velocity. This marked deviation from first-order kinetics indicates an autocatalytic reation mechanism. At the higher oxygen concentration the reaction is first order for approximately four half-lives and the lag phase almost completely eliminated. Reoxidation reactions performed at intermediate oxygen concentrations are autocatalytic with the degree of deviation from first-order kinetics being inversely proportional to the oxygen concentration (data not shown).

The dependence of the linear portion of the semilogarithmic plots on oxygen concentration varies hyperbolically with oxygen concentration (Fig. 2). The most obvious reaction scheme which 

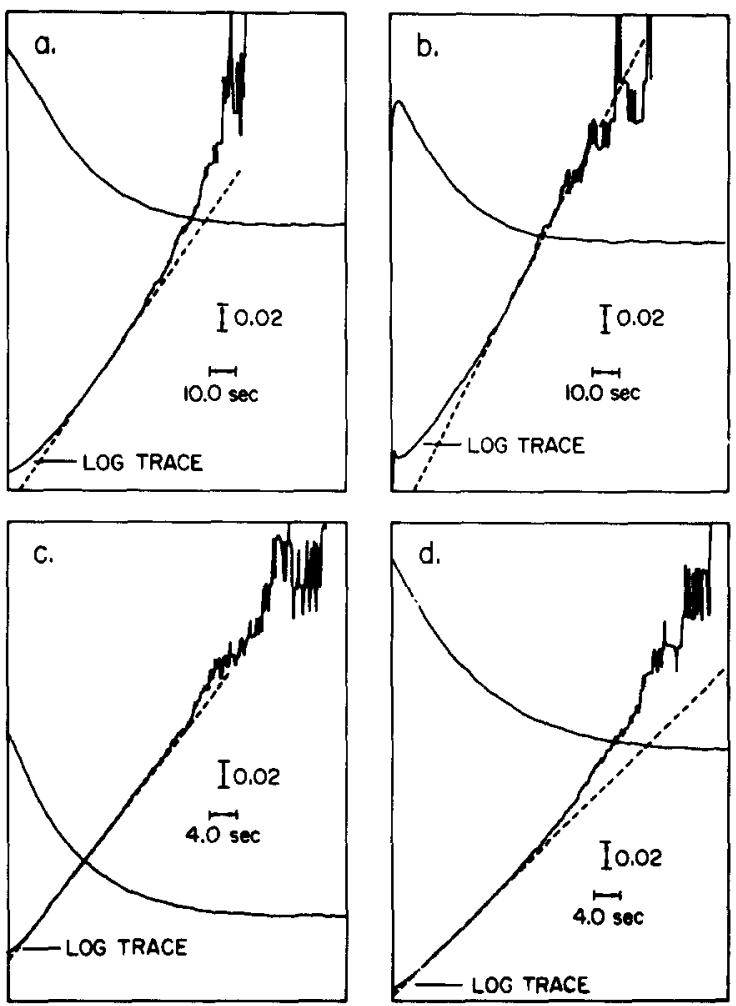

Fig. 1. Stopped-flow trace of reoxidation of $10 \mu \mathrm{M}$ reduced spinach ferredoxin with (a) $0.13 \mathrm{mM}$ oxygen, (b) plus $0.1 \mu \mathrm{M}$ superoxide dismutase, (c) $0.65 \mathrm{mM}$ oxygen, (d) plus $0.1 \mu \mathrm{M}$ superoxide dismutase. The upper, exponential curve is the direct absorbance change at $\mathbf{4 2 0} \mathrm{nm}$ (inverted) versus time. The second trace is the logarithmic plot of the absorbance change. A straight line has been drawn through the logarithmic trace to emphasize any deviation from linearity. The reactions were performed in $0.15 \mathrm{M}$ Tris- $\mathrm{HCl}, \mathrm{pH} 7.3$ at $25^{\circ} \mathrm{C}$. Vertical axis is in absorbance units as shown by indicator. Time increases from left to right on $x$-axis.

will give rise to this hyperbolic dependence is a two-step mechanism with formation of a complex between reduced ferredoxin ( $F d)$ and oxygen (Eqns. 1 and 2) [15]:

$\mathrm{Fd}(\mathrm{II})+\mathrm{O}_{2} \stackrel{k_{1}}{\rightleftharpoons} \mathrm{Fd}(\mathrm{II}) \ldots \mathrm{O}_{2}$

$\mathrm{Fd}(\mathrm{II}) \ldots \mathrm{O}_{2} \stackrel{k_{2}}{\rightleftharpoons} \mathrm{Fd}(\mathrm{III})-\mathrm{O}_{2}^{-}$

The alternate scheme, in which reduced ferredoxin first isomerizes into an activated state and then reacts with oxygen, can be ruled out because the

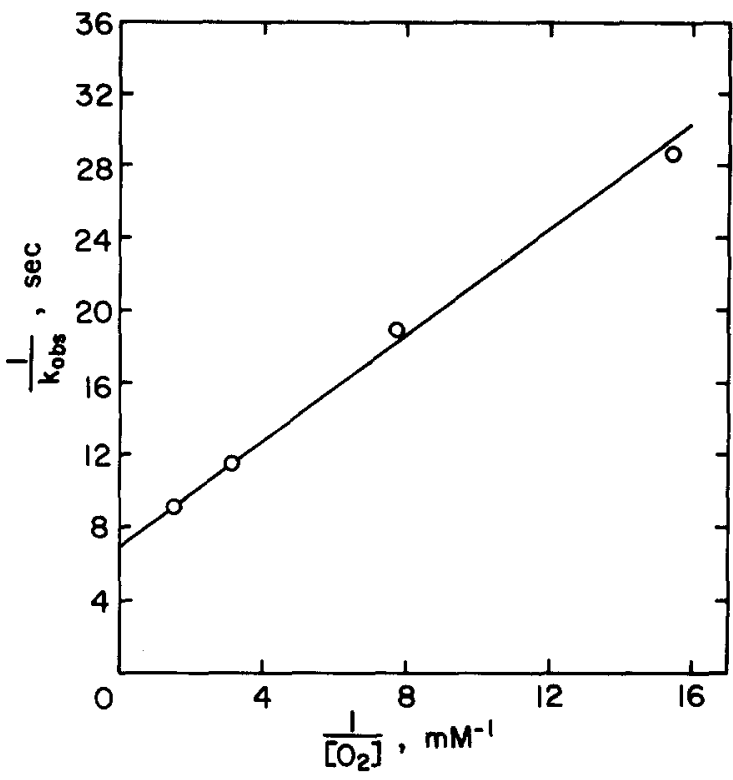

Fig. 2. The rate of reaction of $10 \mu \mathrm{M}$ reduced ferredoxin with oxygen. The data are plotted as the reciprocal of the observed first-order rate constants versus the reciprocal of oxygen concentration which varied from $65 \mu \mathrm{M}$ to $0.65 \mathrm{mM}$. Other details are given in Fig. 1. The correlation coefficient of the individual data points is 0.998 .

addition of saturating oxygen concentrations does not lead to a burst in the production of oxidized ferredoxin followed by the remainder of the reaction proceeding at the slower rate [15]. The lag phase observed at low oxygen concentrations is attributable to the fact that one cannot begin to observe the typical progress of the forward reaction until the ferredoxin-oxygen complex has formed to the steady-state level, and the observation of a lag implies that the rates of the first and second steps approach one another [15].

The linearity of the double-reciprocal plot (Fig. 2) indicates that $k_{-2}$ is negligibly small [15]. This conclusion was verified experimentally as follows. A limiting amount of sodium dithionite $(50 \mu \mathrm{M})$ was reacted with a solution of $28 \mu \mathrm{M}$ oxidized ferredoxin and $130 \mu \mathrm{M}$ oxygen to generate $\mathrm{O}_{2}^{-}$ [14]. Since the rate of reduction of oxygen by dithionite is much faster than the rate of reduction of ferredoxin [14], the reaction produces predominantly $\mathrm{O}_{2}^{-}$. No measurable reduction of ferredoxin was observed, implying that superoxide anion is not able to reduce ferredoxin at a rate comparable 
to that of the disproportionation of $\mathrm{O}_{2}^{-}$or of the rexoidation of reduced ferredoxin by oxygen. This conclusion is to be expected from the relative reduction potentials of $\mathrm{O}_{2}^{-}$and ferredoxin. Using the method of Strickland et al. [15], we thus deduce from the reciprocal of the intercept in Fig. 2 a value of $0.14 \mathrm{~s}^{-1}$ for $k_{2}$. The ratio of the slope to intercept yields a value of $0.19 \mathrm{mM}$ for $K_{\mathrm{d}}$.

The autocatalytic character of the kinetic traces reveals that a product or products of the reaction are also reoxidizing reduced ferredoxin. To test whether $\mathrm{O}_{2}^{-}$could be an oxidant under these conditions, a catalytic amount of superoxide dismutase was added to the oxygen mixtures. Fig. 1c and d) shows that in the presence of superoxide dismutase, the reaction could best be described as biphasic rather than autocatalytic. However, no systematic effect of this reagent was observed. At low oxygen concentrations, the presence of superoxide dismutase appears to enhance the lag phase but makes the later phase of the reaction more exponential in character. At the high oxygen concentrations where the lag phase is minimal, the presence of superoxide dismutase slightly reduces the slope of the semilogarithmic plot at early times and exaggerates the deviations from first-order behavior as the reaction proceeded. However, no noteworthy change in rate was observed at either low or high oxygen concentration.

Since hydrogen peroxide is produced by both the spontaneous and enzymic dismutation of superoxide, the reaction of reduced ferredoxin with $\mathrm{H}_{2} \mathrm{O}_{2}$ was tested directly. The reaction of reduced ferredoxin with hydrogen peroxide is biphasic with essentially no lag. The shape of the semilogarithmic traces varied depending on the peroxide concentration (Fig. 3). Reactions with low concentrations $(0.15 \mathrm{mM})$ of peroxide showed an increase in velocity as the reaction proceeded. At $0.5 \mathrm{mM}$ peroxide the trace was first-order for five half-lives but at $1 \mathrm{mM}$ peroxide the second phase of the reaction was slower than the initial phase. The velocity of the reaction between $\mathrm{H}_{2} \mathrm{O}_{2}$ and reduced ferredoxin was nearly 10-times faster than that of the comparable reaction of oxygen with reduced ferredoxin. At $0.01 \mathrm{mM}$ oxidant the half-life of the reoxidation reaction was $13 \mathrm{~s}$ for oxygen and $1.1 \mathrm{~s}$ for peroxide. At $0.07 \mathrm{mM}$ oxidant the half-life was $6.3 \mathrm{~s}$ for oxygen compared with $0.6 \mathrm{~s}$ for peroxide.
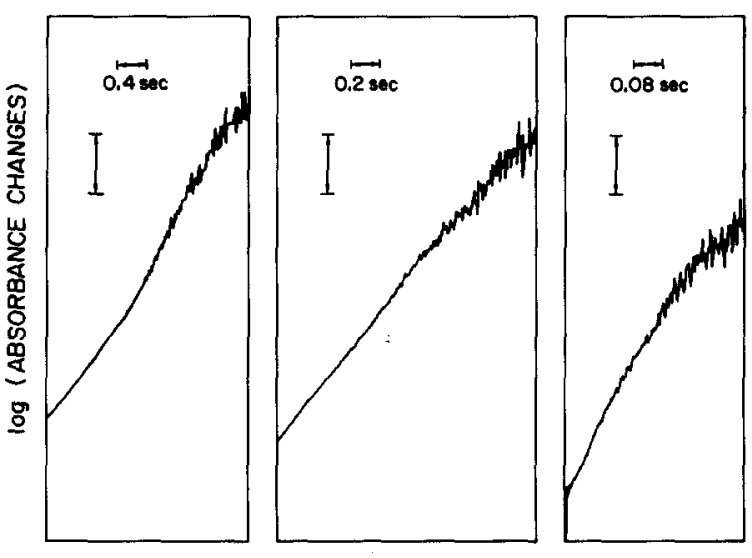

TIME

Fig. 3. The reoxidation of $10 \mu \mathrm{M}$ reduced ferredoxin with three concentrations of hydrogen peroxide. (a) $\left[\mathrm{H}_{2} \mathrm{O}_{2}\right]=0.15 \mathrm{mM}$. (b) $\left[\mathrm{H}_{2} \mathrm{O}_{2}\right]=0.5 \mathrm{mM}$. (c) $\left[\mathrm{H}_{2} \mathrm{O}_{2}\right]=1.0 \mathrm{mM}$. Other conditions as in Fig. 1.

A plot of the initial first-order phase of the reaction versus peroxide concentration (Fig. 4) was linear with a finite intercept, indicative of a simple one-step reversible equilibrium reaction with no significant intermediate (Eqn. 3):

$\mathrm{H}_{2} \mathrm{O}_{2}+\mathrm{Fd}(\mathrm{II}) \underset{k_{-4}}{\stackrel{k_{4}}{\rightleftharpoons}} \mathrm{Fd}(\mathrm{III})+\mathrm{HO}^{*}+\mathrm{OH}^{-}$

The slope and intercept in Fig. 4 yield values of $5.2 \cdot 10^{3} \mathrm{M}^{-1} \cdot \mathrm{s}^{-1}$ and $0.22 \mathrm{~s}^{-1}$ for $k_{4}$ and $k_{-4}$, respectively [15],

It is thus to be expected that hydrogen peroxide will contribute to the overall reaction of ferredoxin both in the presence and absence of dismutase, since it is a product of reaction of reduced ferredoxin with superoxide anion and the dismutation of superoxide anion. We found that hydrogen peroxide is not as effective in reacting with reduced ferredoxin which is already complexed with oxygen, since a $10 \mathrm{mM}$ solution of peroxide in saturating oxygen solution oxidized ferredoxin with a half-time of $1.5 \mathrm{~s}$, a rate which could be obtained with $0.25 \mathrm{mM}$ peroxide in anaerobic solution. This indicates that the contribution of peroxide to the overall reaction at saturating oxygen concentrations is limited. The contribution of $\mathrm{H}_{2} \mathrm{O}_{2}$ to the overall reaction could not be determined by adding catalase because catalase enhances the rate of reoxidation, although it does not oxidize ferred- 


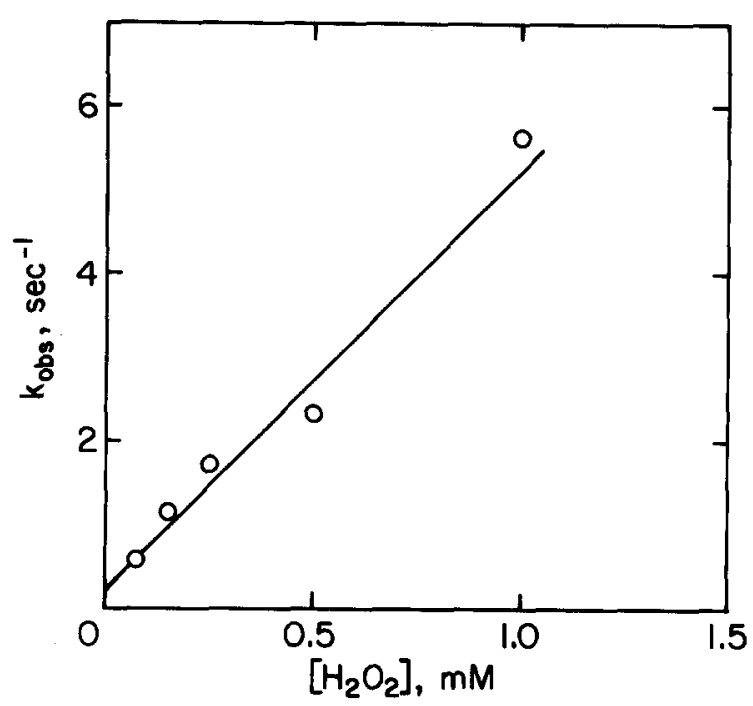

Fig.4. The dependence of $\boldsymbol{k}_{\text {obs }}$ on the concentration of hydrogen peroxide for the reaction of $10 \mu \mathrm{M}$ reduced ferredoxin with hydrogen peroxide, the concentration of which varied from 0.075 to $1.0 \mathrm{mM}$. Other conditions as in Fig. 1. The correlation coefficient of the individual data points is $\mathbf{0 . 9 9 0}$.

oxin anaerobically (data not shown). The increase in velocity is especially significant because catalase concomitantly destroys any peroxide formed in the reaction. In addition, in the presence of catalase the reaction conforms more nearly to first-order kinetics (in the case of $0.65 \mathrm{mM} \mathrm{O}_{2}$ ), probably because the autocatalytic effect of peroxide is eliminated. The catalysis of reoxidation of ferredoxin by catalase is not due to its peroxidative activity, because large excesses of ethanol do not inhibit the effect. Horseradish peroxidase did not affect the reoxidation rate or kinetics.

A related class of reaction has been extensively studied for the oxidation of certain divalent transition metal ions and the following reaction mechanism has been established [16]:

$\mathrm{M}(\mathrm{II})+\mathrm{H}_{2} \mathrm{O}_{2}=\mathrm{M}(\mathrm{III})+\mathrm{HO}^{\circ}+\mathrm{OH}^{-}$

$\mathrm{M}(\mathrm{II})+\mathrm{HO}^{\circ} \rightleftharpoons \mathrm{M}(\mathrm{III})+\mathrm{OH}^{-}$

To test whether radicals formed during the reaction were responsible for the second phase of the reaction, $0.05 \mathrm{M}$ sodium formate was added as a radical trapper to the reaction mixtures at three representative concentrations of peroxide. For-

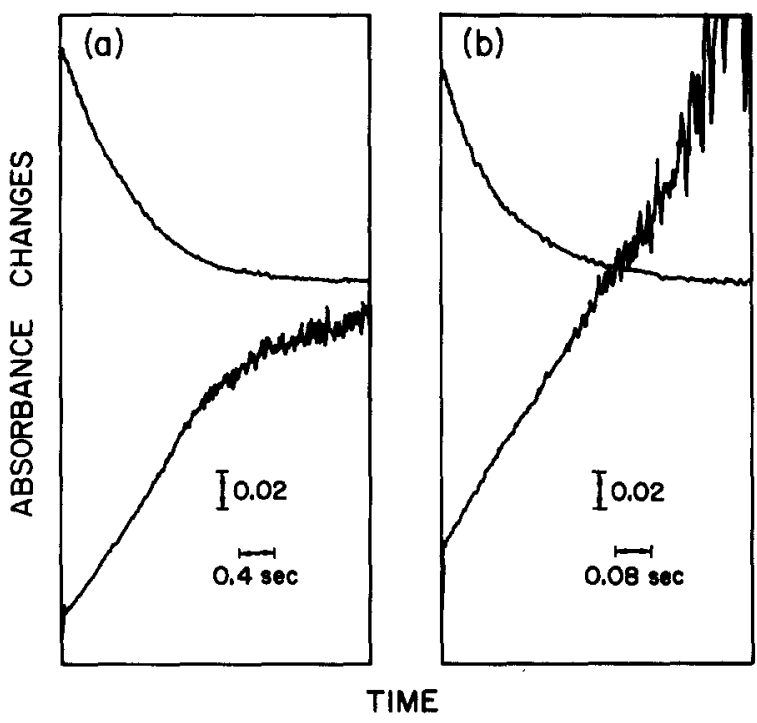

Fig. 5. The reoxidation of $10 \mu \mathrm{M}$ reduced ferredoxin with hydrogen peroxide in the presence of $0.05 \mathrm{M}$ sodium formate. (a) $\left[\mathrm{H}_{2} \mathrm{O}_{2}\right]=0.15 \mathrm{mM}$. (b) $\left[\mathrm{H}_{2} \mathrm{O}_{2}\right]=1.0 \mathrm{mM}$. Other conditions as in Fig. 1.

mate eliminated the acceleration of the second phase of the reaction at low peroxide concentration, eliminated the slower second phase of the reaction at high peroxide concentration, but did not alter the initial rates of either reaction (Fig. 5). The conclusion is thus drawn that the second phase of the reaction is mediated by radicals formed during the reaction of ferredoxin with peroxide, but the first phase is not.

\section{Discussion}

The most unexpected finding of this study is represented in Eqn. 3, that reduced ferredoxin reacts with hydrogen peroxide directly. The reduction of hydrogen peroxide by transition metal ions with the production of hydroxyl radical [16] has been shown to occur with several inorganic systems [16] and is typified by Fenton's reagent, hydrogen peroxide and a ferrous salt [17], which is used as a powerful oxidant of organic substrates. The first step of the resulting complex series of reactions is:

$\mathrm{Fe}(\mathrm{II})+\mathrm{H}_{2} \mathrm{O}_{2} \rightleftharpoons \mathrm{Fe}(\mathrm{III})+\mathrm{OH}^{-}+\mathrm{HO}^{\circ}\left(k=76 \mathrm{M}^{-1} \cdot \mathrm{s}^{-1}\right)$ 
followed by:

$\mathrm{HO}^{\circ}+\mathrm{Fe}(\mathrm{II}) \rightleftharpoons \mathrm{Fe}(\mathrm{III})+\mathrm{OH}^{-}\left(k=3 \cdot 10^{8} \mathrm{M}^{-1} \cdot \mathrm{s}^{-1}[17]\right)$

To compare the rate of the observed reaction of reduced ferredoxin with $\mathrm{H}_{2} \mathrm{O}_{2}$ and the rate of the first step of Fenton s reagent, calculations were made based on the reaction of $10 \mu \mathrm{M}$ ferredoxin with $10 \mu \mathrm{M} \mathrm{H}_{2} \mathrm{O}_{2}$ which had a half-time of $1.1 \mathrm{~s}$. The rate constant for the reaction of Fenton's reagent would predict a velocity of $7.6 \cdot 10^{-9} \mathrm{M}^{-1}$ $\cdot \mathrm{s}^{-1}$, whereas the velocity observed in the ferredoxin system was $10^{3}$-times faster, or $5 \cdot 10^{-6}$ $\mathbf{M}^{-1} \cdot \mathrm{s}^{-1}$. The conditions for the ferredoxin reaction are also much milder as Fenton's reagent is typically used with perchlorate salts at $\mathrm{pH}<2$ [17].

The second step in the inorganic systems is believed to be the oxidation of the divalent transition metal by hydroxyl radical. The anticipated rates for a hydroxyl radical-mediated reaction with ferredoxin coincide with the observed rates only if very low amounts of radical are generated. Alternatively, $\mathrm{HO}^{-}$generated by reduction of $\mathrm{H}_{2} \mathrm{O}_{2}$ may react with excess $\mathrm{H}_{2} \mathrm{O}_{2}$ to form superoxide anion [18], which then secondarily reacts with reduced ferredoxin, but at a slower rate than either peroxide or hydroxyl radical. The reaction of peroxide with hydroxyl radical would therefore be a quenching reaction, and the effective concentration of hydroxyl radical in solution would be much less than the amount generated from ferredoxin reoxidation. As the peroxide concentration is increased, the quenching effect would also increase, and the second phase reaction would not increase in rate, which is in agreement with the experimental findings. The observed decrease in the rate of the second phase of the peroxide reaction at the highest peroxide concentration may be due to denaturation of the enzyme, as has been observed for superoxide dismutase [19].

Whereas reduced ferredoxin reacts with hydrogen peroxide in a one-step equilibrium reaction with no signifiant intermediate, reoxidation with oxygen as electron donor proceeds by way of complex formation. The nature of this reduced ferredoxin-oxygen complex is probably charge transfer rather than covalent with outer-sphere electron transport expected. X-ray analysis of the structure of the $[\mathrm{Fe}-\mathrm{S}]_{2}$ protein from Spirulina platensis [20] shows that the most likely mode of reaction proceeds via the orbitals of cysteinyl sulfur from residues 39 and 44 which are located on the surface of the molecule. The ferrous iron constituent of the binuclear cluster has tetrahedral symmetry $[21,22]$, but data on the coordination number and symmetry of the ferric component are not available for either oxidized or reduced states. However, there is a report that the ferric sites in oxidized spinach ferredoxin are inequivalent, and the nonreducible site in reduced ferredoxin is proposed to be a highly distorted (squashed toward $D_{2 d}$ ) tetrahedral structure with the possibility of an additional weak coordination to an available nitrogen or oxygen donor atom [23]. Conceivably, oxygen can distort or partially displace one of the sulfur ligands and form a complex with the iron atom bonded by Cys-39 and Cys-44.

The ability to form an adduct with the active site is a property unique to oxygen. the ferredoxin chromophore is unstable to the addition of a related ligand, nitric oxide (NO) which is smaller than molecular oxygen and differs in electronic structure by having one less electron. NO reacts with heme proteins by donating an electron to the iron-porphyrin ring system, creating new EPR signals and making possible the identification of iron ligands [24]. In contrast, reaction of NO with oxidized ferredoxin, under conditions which eliminate the possibility of acid denaturation due to nitric acid formation, results in destruction of the iron-sulfur chromophore, as monitored by the loss in optical absorption and visible circular dichroism [25]. Thus, the geometry of the active center of ferredoxin cannot tolerate the addition of a nitrosyl ligand.

The production of superoxide in this system was established by both Misra and Fridovich [5] and Nakamura and Kimura [7], who measured production of $\mathrm{O}_{2}^{-}$directly by coupling superoxide anion to the oxidation of epinephrine. Earlier attempts at detecting superoxide anion by magnetic resonance had been successful for bacterial ferredoxin, but not for plant ferredoxin [26]. Because bacterial ferredoxin reacts more rapidly with oxygen than does spinach ferredoxin, and the EPR method can only monitor steady-state levels, the total yield of radical formed is below detectability 
with the latter system. Not only would the superoxide anion produced in the reaction continually dismute, but any direct reaction of the superoxide anion with reduced ferredoxin would also lower its steady-state concentration. According to our measurements, only $0.3-6 \%$ of the reaction would have taken place at the points monitored by EPR; consequently, the superoxide anion produced would have been below the level of detection.

The small but real changes that we observe in the presence of superoxide dismutase suggest that either $\mathrm{O}_{2}^{-}$also acts as an oxidant in the overall reaction or that the enzyme changes the kinetic pattern by accelerating production of hydrogen peroxide. The slight but reproducible inhibitory effect of superoxide dismutase on the initial rate of reoxidation of ferredoxin supports the original observation of Allen [10]. The difference in the magnitude of the inhibitory effect which we detected (approx. 10\% compared with 25-40\% reported by Allen [10]) may be attributed to the large excess of ferredoxin present in his system. With ferredoxin in excess, superoxide formed may react rapidly with ferredoxin not complexed with oxygen and may therefore contribute more to the overall rate of reoxidation than it does under our conditions. The finding that superoxide anion might oxidize reduced ferredoxin throws light on the finding of Nakamura and Kimura [7] that interaction with cytochrome $c$ stimulates the oxidase activity of reduced ferredoxin 20 -fold, but stimulates the production of superoxide anion, as measured by oxidation of epinephrine and sulfite, only 6-10fold. A portion of the superoxide generated in their system may have been further reduced to $\mathrm{H}_{2} \mathrm{O}_{2}$ by reduced ferredoxin at a rate comparable to that of epinephrine oxidation.

The reoxidation rate of spinach ferredoxin is of the same order of magnitude as that of flavins and xanthine oxidase $[9,27]$, though it is much slower than the electron-transfer process to ferredoxin$\mathrm{NADP}^{+}$reductase and $\mathrm{NADP}^{+}$. Conversely, the rate of reoxidation of ferredoxin is much faster than that of air oxidation of $\mathrm{Fe}^{2+}$ in solution.

Is there a physiological significance in the ability of reduced ferredoxin to react with oxygen, superoxide anion, and peroxide? It has been shown that isolated chloroplasts generate $\mathrm{O}_{2}^{-}$on illumination [28]. Isolated chloroplasts have also been shown to produce hydrogen peroxide by monovalent and divalent reduction of oxygen $[29,30]$, and peroxide inhibits photosynthesis by $50 \%$ at concentrations as low as $10^{-5} \mathrm{M}$ [31]. Although some catalase activity is usually found with preparations of isolated chloroplasts, it has been suggested that this activity is derived from catalase which was originally located in peroxisomes and is liberated upon homogenization of the leaves [32]. In addition, catalase is inhibited by ascorbate [33], and ascorbate is found in chloroplasts at concentrations up to $50 \mathrm{mM}$ [34]. The importance of catalase, if present in chloroplasts, in the destruction of hydrogen peroxide in chloroplasts therefore should only be very small. Since hydrogen peroxide, superoxide anion and hydroxyl radical have been implicated in the molecular mechanisms for cytotoxicity [35], the ability of reduced ferredoxin to reduce these products to water may play a protective role in plant physiology.

\section{Acknowledgements}

This research was supported by Grants GM 12176 and GM 21337 from the National Institutes of Health and C636 from the Welch Foundation.

\section{References}

1 Arnon, D.I., Tsujimoto, H.Y. and McSwain, B.D. (1964) Proc. Natl. Acad. Sci. U.S.A. 51, 1274-1282

2 Whatley, F.R., Tagawa, K. and Arnon, D.I. (1963) Proc. Natl. Acad. Sci. U.S.A. 49, 266-270

3 Arnon, D.I., Tsujimoto, H.Y. and McSwain, B.D. (1967) Nature 214, 562-566

4 Telfer, A., Cammack, R. and Evans, M.C.W. (1970) FEBS Lett. 10, 21-24

5 Misra, H.P. and Fridovich, I. (1971) J. Biol. Chem. 246, 6886-6890

6 Foust, G.P., Mayhew, S.G. and Massey, V. (1969) J. Biol. Chem. 244, 964-970

7 Nakamura, S. and Kimura, T. (1972) J. Biol. Chem. 247, 6462-6468

8 Olson, J.S., Ballou, D.B., Palmer, G. and Massey, V. (1974) J. Biol. Chem. 249, 4364-4382

9 Porras, A.G., Olson, J.S. and Palmer, G. (1981) J. Biol. Chem. 256, 9090-9103

10 Allen, J.F. (1975) Biochem. Biophys. Res. Commun. 66, 36-43

11 Halliwell, B. (1974) New Phytol. 73, 1075-1086

12 Petering, D.H. and Palmer, G. (1970) Arch. Biochem. Biophys. 141, 456-464 
13 Ballou, D.P. (1971) Ph.D. Dissertation, University of Michigan, Ann Arbor

14 Lambeth, D.O. and Palmer, G. (1973) J. Biol. Chem. 248, 6095-6103

15 Strickland, S., Palmer, G. and Massey, V. (1975) J. Biol. Chem. 250, 4048-4052

16 Dixon, W.T. and Norman, R.O.C. (1962) Nature 196, 891-892

17 Walling, C. (1975) Acc. Chem. Res. 8, 125-131

18 Symonyn, M.A. and Nalbandyan, R.M. (1979) Biochim. Biophys. Acta 583, 279-286

19 Bray, R.C., Cockle, S.A., Fielden, E.M., Roberts, P.B., Rotilio, G. and Calabrese, L. (1974) Biochem. J. 139, 43-48

20 Fukuyama, K., Hase, T., Matsumoto, S., Tsukihara, T., Katsube, Y., Tanaka, N., Kakudo, M., Wada, K. and Matsubara, H. (1980) Nature 286, 522-524

21 Eaton, W.A., Palmer, G., Fee, J.A., Kimura, T. and Lovenberg, W. (1971) Proc. Natl. Acad. Sci. U.S.A. 68, 3015-3020

22 Hosein, B., Friesner, R. and Holzwarth, G. (1974) Biochim. Biophys. Acta 368, 19-21

23 Rawlings, J., Siiman, O. and Gray, H.B. (1974) Proc. Natl; Acad. Sci. U.S.A. 71, 125-127
24 Yonetani, T., Yamamoto, H., Erman, J.E., Leigh, J.S. and Reed, G.H. (1972) J. Biol. Chem. 247, 2447-2455

25 Hosein, B. (1973) Ph.D. Thesis, University Microfilms, University of Michigan, Ann Arbor

26 Orme-Johnson, W.H. and Beinert, H. (1969) Biochem. Biophys. Res. Commun. 36, 905-911

27 Massey, V., Palmer, G. and Ballou, D. (1973) in Oxidases and Related Redox Systems, Vol.1 (King, T.E., Mason, H.S. and Morrison, M., eds.), pp. 25-50, University Park Press, Baltimore, MD

28 Asada, K. and Kiso, K. (1973) Agric. Biol. Chem. 37, 453

29 Epel, B.L. and Neumann, J. (1973) Biochim. Biophys. Acta $325,520-529$

30 Elstner, E.F. and Frommeyer, D. (1978) FEBS Lett. 86, 143-146

31 Kaiser, W. (1976) Biochim. Biophys. Acta 440, 476-482

32 Allen, J.F. and Whatley, F.R. (1978) Plant Physiol. 61, 957-960

33 Orr, C.W.M. (1967) Biochemistry 6, 2995-2999

34 Halliwell, B. (1978) Prog. Biophys. Mol. Biol. 33, 1-54

35 Cohen, G. and Heikkila, R.E. (1974) J. Biol. Chem. 249, 2447-2452 